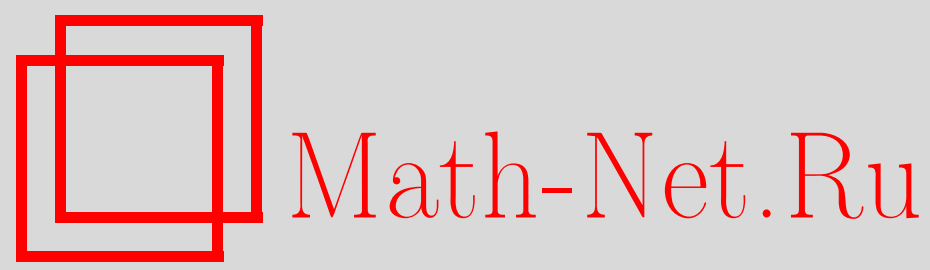

Х. К. Ишкин, Критерий расщепления спектра, Матем. заметки, 2002, том 72, выпуск 5, 670-681

DOI: https://doi.org/10.4213/mzm455

Использование Общероссийского математического портала Math-Net.Ru подразумевает, что вы прочитали и согласны с пользовательским соглашением http://www.mathnet.ru/rus/agreement

Параметры загрузки:

IP : 54.198 .187 .58

26 апреля 2023 г., $11: 22: 26$

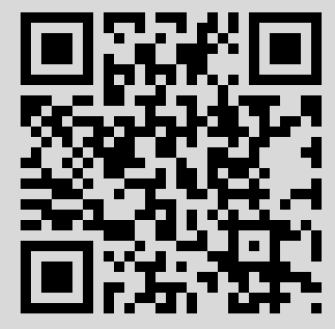


УДК 517.927.25

\section{КРИТЕРИЙ РАСЩЕПЛЕНИЯ СПЕКТРА}

\section{Х. К. Ишкин}

В работе получено необходимое и достаточное условие расщепления спектра для произвольного несимметричного потенциала, наименьшее значение которого достигается в конечном чис ле точек.

Библиограффия: 6 названий.

1. Формулировка основного результата. Рассмотрим задачу Штурма-Лиувилля

$$
\begin{gathered}
l(k) y=-k^{-2} y^{\prime \prime}+q(x) y=\lambda y, \quad x \in(0 ; 1) \\
y(0)=y(1)=0 .
\end{gathered}
$$

Здесь $k$ - положительньй параметр, $q$ - вещественнозначная, дважды непрерьвно дифференцируемая на $[0 ; 1]$ функция.

В работе [1] доказано, что если $q(x)$ - четная дважды непрерьвно дифференцируемая на $[-1 ; 1]$ функция и имеет изолированньй локальньй максимум в точке $x=0$ (потенциальньй барьер), то собственные значения задачи (1)-(2), расположенные ниже потенциального барьера, группируются парами $\lambda_{n}^{+}$и $\lambda_{n}^{-}$, причем их разность при $k \rightarrow \infty$ экспоненциально мала - происходит так называемое расщепление спектра. Метод этой работы существенно связан с четностью потенциала.

В предлагаемой работе получено необходимое и достаточное условие расщепления спектра для произвольного несимметричного потенциала, наименьшее значение которого достигается в конечном числе точек.

Введем обозначения. Без ограничения общности можно считать, что наименьшее значение функции $q(x)$ равно 0 . Пусть функция $q(x)$ обращается в нуль в точках $0 \leqslant a_{1}<$ $\cdots<a_{n} \leqslant 1$ и положительна вне этих точек. В дальнейшем при выполнении этих условий будем говорить, что $q$ имеет $n$ потенциальных ям. Пусть $b_{i} \in\left(a_{i} ; a_{i+1}\right)(i=1, \ldots, n)$, $b_{0}=0, b_{n+1}=1$. Обозначим через $L_{i}(k)$ самосопряженные операторы, порожденные в $L^{2}\left[b_{i} ; b_{i+1}\right]$ дифференциальным выражением $l(k)$ и краевыми условиями $y\left(b_{i}\right)=$ $y\left(b_{i+1}\right)=0, i=1, \ldots, n$. Через $L(k)$ обозначим оператор, соответствующий задаче (1)-(2). Далее, пусть $\left\{\lambda_{j}\right\}_{j=1}^{\infty}$ и $\left\{\mu_{j}^{i}\right\}_{j=1}^{\infty}-$ собственные значения операторов $L(k)$ и $L_{i}(k)$, пронумерованные в порядке возрастания. 
ТЕОРемА 1. Пусть функиия $q$ дважды непрерывно дифференцируема на [0;1] u имеет $n$ потенциальных ям. Тогда оператор $L(k)$ имеет $n$ әкспоненциально близких при $k \rightarrow \infty$ собственных значений $\lambda_{m}(k), \ldots \lambda_{m+n-1}(k)$, m.е. $\lambda_{i}(k)-\lambda_{j}(k)=$ $O\left(e^{-\delta k}\right), i, j=m, \ldots, m+n-1$, с некоторым $\delta>0$, тогда и только тогда, когда $у$ операторов $L_{1}(k), \ldots, L_{n}(k)$ существуют собственные значения $\mu_{j_{1}}^{1}(k), \ldots, \mu_{j_{n}}^{n}(k)$, где $j_{1}, \ldots, j_{n}$ не зависят от $k$, также әкспоненциально близкие при $k \rightarrow \infty$.

Следующие 2 пункта подготавливают доказательство теоремы, которое будет дано в п. 4. При этом в пш. 2 и 3 попутно устанавливаются и другие свойства оператора $L(k)$, представляющие самостоятельный интерес.

2. Некоторые свойства оператора $L(k)$ с одной потенциальной ямой. В этом пункте мы покажем, что экспоненциально малое расщепление возможно лишь при наличии более одной ямы.

ТЕОРема 2. Пусть $q$ имеет одну потенииальную яму и пусть $\lambda_{1}(k), \lambda_{2}(k), \ldots$ собственные значения оператора $L(k)$, занумерованные в порядке возрастания. Тогда при любом фиксированном $i \in \mathbb{N}$

$$
\left(\lambda_{i+1}(k)-\lambda_{i}(k)\right) e^{\delta k} \rightarrow \infty, \quad k \rightarrow \infty
$$

для всякого положительного $\delta$.

Доказательство теоремы будет разбито на несколько лемм. Но прежде мы докажем один результат, справедливый для произвольного (даже бесконечного) числа ям.

Лемма 1. а) Пусть q непрерывна на $[0 ; 1]$. Тогда $k^{2} \cdot \lambda_{i}(k)$ и соответствующая нормированная собственная функиия $f_{i}(x, k)$ при каждом фиксированном $x \in[0 ; 1]$ аналитичны в некоторой окрестности вещественной прямой. При $k \rightarrow \infty \lambda_{i}(k)$ монотонно убывает до 0.

б) Если $q \in C^{2}[0 ; 1]$ и существует точка $a \in[0 ; 1]$ такая, что $q(a)=q^{\prime}(a)=0$, mо $\lambda_{i}(k)=O\left(k^{-1}\right), k \rightarrow \infty$. Eсли же такой точки не существует ${ }^{1}$, то $\lambda_{i}(k)=$ $O\left(k^{-2 / 3}\right)$.

ДокАЗАТЕЛЬСТво. а) Положим $M(k)=k^{2} L(k)$. Тогда $M(k)=L_{0}+k^{2} Q$, где $L_{0}$ - дифференциальный оператор, порожденный в $L^{2}[0 ; 1]$ дифференциальным выражением $-d^{2} / d x^{2}$ и краевыми условиями $(2), Q$ - оператор умножения на функцию $q$. Так как $Q$ бесконечно мал относительно $L_{0}$, то $M(k)$ - целое семейство в смысле Като [2, $\S \mathrm{XII.2,} \mathrm{лемма} \mathrm{2].} \mathrm{Отсюда} \mathrm{в} \mathrm{силу} \mathrm{невырожденности} \mu_{i}(k)($ собственных значений $M(k))$ при каждом $k>0$ по теореме XII.9 из [2] заключаем, что $\mu_{i}(k)$ и $f_{i}(x, k)$ при фиксированных $x \in[0 ; 1]$ аналитичны в некоторой окрестности вещественной прямой. Но $\lambda_{i}(k)=k^{-2} \mu_{i}(k)$, откуда следует первая часть утверждения а).

То, что $\lambda_{i}(k)$ убывают, следует из того, что семейство операторов $L(k)=k^{-2} L_{0}+Q$ убывает на $(0 ; \infty)$. Далее, если

$$
\lim _{k \rightarrow \infty} \lambda_{i}(k)=\lambda_{0}>0
$$

\footnotetext{
${ }^{1}$ В этом случае $q$ имеет одну или две точки минимума на концах отрезка $[0 ; 1]$.
} 
то найдется некоторый отрезок, содержащий точку минимума функции $q$, в котором в соответствии с теоремой сравнения Штурма (см., [3, гл. XI, теорема 3.1$])$ функция $f_{i}(x, k)$ имеет неограниченное по $k$ число нулей, что невозможно.

б) Если $q(a)=q^{\prime}(a)=0$, то найдется некоторое положительное число $\alpha$ такое, что $q(x) \leqslant \alpha^{2}(x-a)^{2}$ для всех $x \in[0 ; 1]$. Пусть $H(k)$ - оператор, которьй получается из $L(k)$ заменой $q$ на $\alpha^{2}(x-a)^{2}$. Поскольку $L(k) \leqslant H(k)$, то $\lambda_{i}(k) \leqslant \mu_{i}(k), i=1,2, \ldots$, где $\mu_{i}(k)$ - собственные значения оператора $H(k)$. Докажем, что $\mu_{i}(k)=O\left(k^{-1}\right), k \rightarrow \infty$.

Рассмотрим сначала случай $a \in(0 ; 1)$. Введем в рассмотрение гармонический осциллятор $H_{\infty}(k)$, порожденный в $L^{2}(-\infty ;+\infty)$ дифференциальньм выражением $k^{-2} d^{2} / d x^{2}$ $+\alpha^{2}(x-a)^{2}$. Собственные значения $H_{\infty}(k)$ вычисляются точно (см., например, [4, $§ 24$, п. $6($ д)] $): \nu_{i}(k)=(2 i+1) \alpha / k$, а соответствуюшие нормированные собственные функции имеют вид

$$
\psi_{i}(x, k)=C_{i} e^{-\alpha k(x-a)^{2} / 2} H_{i}(\sqrt{\alpha k}(x-a)), \quad C_{i}=\sqrt[4]{\frac{k}{\pi \alpha}} \frac{1}{\sqrt{2^{i} i !}}
$$

где $H_{i}$ - полиномы Эрмита.

Покажем, что для любого $i$ найдутся $c_{i}>0, d_{i}>0$ такие, что для всех $k>0$

$$
\mu_{i}(k) \leqslant \nu_{i}(k)+c_{i} e^{-d_{i} k}
$$

Выберем положительное число $\varepsilon$ так, чтобы $[a-\varepsilon ; a+\varepsilon] \in(0 ; 1)$ и введем гладкую функцию $\chi(x)$, равную 1 на $[a-\varepsilon / 2 ; a+\varepsilon / 2]$ и 0 вне $(a-\varepsilon ; a+\varepsilon)$. Далее положим $\phi_{j}=\psi_{j} \chi /\left\|\psi_{j} \chi\right\|, j=1, \ldots, i$. Тогда $\phi_{j} \in D(H(k)),\left[H(k)-\nu_{j}(k)\right] \phi_{j}=O\left(e^{-d_{i} k}\right)$, $\left(\phi_{l}, \phi_{j}\right)=\delta_{l j}+O\left(e^{-d_{i} k}\right), l, j=1, \ldots, i, k \rightarrow \infty$, равномерно по $x \in[0 ; 1]$ с некоторым $d_{i}>0$. Отсюда, применяя принцип минимакса [2, с. 91], получим (3), что доказьвает лемму при $a \neq 0,1$. Если же $a=0$ или 1 , то в качестве $H_{\infty}(k)$ выберем гармонический осциллятор на полуоси $(0 ; \infty)$ или $(-\infty ; 1]$ с условиями Дирихле в 0 или 1 . Все остальные рассуждения почти дословно совпадают с рассмотренным вьше случаем.

Пусть теперь $q(0)=0, q^{\prime}(0) \neq 0$. Тогда $q(x) \leqslant \alpha x, x \in[0 ; 1]$ при некотором $\alpha>0$. Введя по аналогии с предыдушим оператор $H_{\infty}(k)$, порожденньй в $L^{2}[0 ; \infty)$ дифференциальным выражением $k^{-2} d^{2} / d x^{2}+\alpha x$ и краевьм условием $y(0)=0$, будем иметь

$$
\nu_{i}(k)=\xi_{i} \alpha^{-1 / 3} k^{-2 / 3}, \quad \psi_{i}(x, k)=c_{i} A i\left(k^{2 / 3} \alpha^{1 / 3} x\right)
$$

где $A i(\xi)$ - функция Эйри $\left[5\right.$, гл. IV, п. 2], $-\xi_{i}<0-$ корни функции Эйри. Отсюда, воспользовавшись асимптотикой $[5$, гл. IV,$\S 1$, п. 2.2]

$$
A i(\xi) \sim \frac{1}{2 \sqrt{\pi}} \xi^{-1 / 4} e^{-2 \xi^{3 / 2} / 3}, \quad \xi \rightarrow+\infty
$$

и повторяя рассуждения, проведенные для гармонического осциллятора, получим утверждение леммы.

Обратимся теперь к случаю одной потенщиальной ямы. Итак, пусть $q(a)=0$, $q(x)>0, x \neq a$. Обозначим через $T_{1}(k)$ и $T_{2}(k)$ самую левую и самую правую точки 
поворота, т.е. решения уравнения $q(T)=\lambda(k)^{2}$. Из п. а) леммы 1 следует, что при $k \rightarrow \infty$

$$
T_{1}(k) \rightarrow a-0, \quad T_{2}(k) \rightarrow a+0 .
$$

Обозначим через $u_{i}(x, k)$ и $v_{i}(x, k)$ решения уравнения $(1)$ при $\lambda=\lambda_{i}(k)$, удовлетворяющие начальным условиям

$$
u_{i}\left(T_{1}, k\right)=1, \quad u_{i}^{\prime}\left(T_{1}, k\right)=0, \quad v_{i}\left(T_{1}, k\right)=0, \quad v_{i}^{\prime}\left(T_{1}, k\right)=1 .
$$

ЛЕмма 2. Функиия $u_{i}$ убывает на отрезке $\left[0 ; T_{1}(k)\right]$.

ДокАЗАТЕЛЬСтво. Покажем, что $u_{i}^{\prime}<0$ при всех $x \in\left[0 ; T_{1}(k)\right]$. Имеем

$$
u_{i}(x, k)=1+k^{2} \int_{x}^{T_{1}}(t-x)\left(q(t)-\lambda_{i}(k)\right) u_{i} d t
$$

откуда

$$
u_{i}^{\prime}(x, k)=-k^{2} \int_{x}^{T_{1}}\left(q-\lambda_{i}(k)\right) u_{i} d t .
$$

Так как $u_{i}\left(T_{1}, k\right)=1$, то $u_{i}>0$ в некоторой левой полуокрестности точки $T_{1}$, так что $u_{i}^{\prime}<0$ в этой полуокрестности. Допустим, что существует точка $x_{0}$ из $\left[0 ; T_{1}\right)$, в которой $u_{i}^{\prime}$ обращается в 0 и $u_{i}^{\prime}<0$ на $\left(x_{0} ; T_{1}\right)$. Но тогда $u_{i}>1$ при таких $x ;$ следовательно,

$$
u_{i}^{\prime}\left(x_{0}, k\right)<-k^{2} \int_{x_{0}}^{T_{1}}\left(q-\lambda_{i}(k)\right)<0 .
$$

Лемма 3. Для функиий $u_{i} u v_{i}$ при $k \rightarrow \infty$ справедливы равномерные по $x \in$ $\left[T_{1}(k) ; T_{2}(k)\right]$ асимптотические оченки

$$
u_{i}, v_{i}=O\left(e^{o(k)}\right)
$$

ДокАЗАТЕЛЬСТВо. Из (5) при $x \in\left[T_{1}(k) ; T_{2}(k)\right]$ имеем

$$
\left|u_{i}\right| \leqslant 1+\lambda_{i}(k) k^{2}\left(T_{2}(k)-T_{1}(k)\right) \int_{T_{1}}^{x}\left|u_{i}\right| d t .
$$

Согласно п. б) леммы 1 и (4) $\lambda_{i}(k) k^{2}\left(T_{2}(k)-T_{1}(k)\right)=o(k)$, откуда, итерируя последнее неравенство, получим доказьваемую оценку для $u_{i}$.

Функция $v_{i}$ удовлетворяет уравнению

$$
v_{i}(x, k)=x-T_{1}+k^{2} \int_{x}^{T_{1}}(t-x)\left(q(t)-\lambda_{i}(k)\right) v_{i} d t .
$$

Отсюда, повторяя проведенные вьше рассуждения для $v_{i}$, получим утверждение леммы.

Пусть $f_{i}(x, k)$ - нормированная собственная функция оператора, соответствующая $\lambda_{i}(k)$. Тогда $f_{i}^{\prime}\left(T_{1}, k\right) \neq 0$. Действительно, если бы $f_{i}^{\prime}\left(T_{1}, k\right)=0$, то $f_{i}=c u_{i}$ с некоторой постоянной $c \neq 0$. Тогда $f_{i}(0, k) \neq 0$, что невозможно. Без ограничения общности можно считать, что $f_{i}^{\prime}\left(T_{1}, k\right)>0$.

\footnotetext{
${ }^{2}$ Здесь и далее в этом пункте считаем, что $a \in(0 ; 1)$. В случае, когда $a$ совпадает с одним из концов отрезка $[0 ; 1]$, все выкладки сохраняют силу.
} 
ЛЕмма 4. При указанной нормировке функиия $f_{i}(x, k)$ возрастает на $\left[0 ; T_{1}(k)\right]$ и для всех $x \in\left[0 ; T_{1}(k)\right] f_{i}(x, k) u_{i}(x, k)<f_{i}^{\prime}\left(T_{1}, k\right)$.

ДокАЗАТЕЛЬСТво. Имеем

$$
\left(\frac{f_{i}}{u_{i}}\right)^{\prime}=\frac{W\left(u_{i}, f_{i}\right)}{u_{i}^{2}}=\frac{f_{i}^{\prime}\left(T_{1}, k\right)}{u_{i}^{2}} .
$$

Интегрируя это равенство по $[0 ; x]$, получим

$$
f_{i}(x, k)=f_{i}^{\prime}\left(T_{1}, k\right) u_{i}(x, k) \int_{0}^{x} u_{i}^{-2} d t
$$

Отсюда согласно лемме 2 следует, что $f_{i}$ положительна на $\left(0 ; T_{1}\right]$, так что $f_{i}^{\prime}(0, k)>0$. Но $f_{i}^{\prime \prime}=k^{2}\left(q-\lambda_{i}(k)\right) f_{i}$; следовательно, $f_{i}^{\prime \prime}>0$ на $\left(0 ; T_{1}\right]$. Значит, $f_{i}^{\prime}$ возрастает на $\left(0 ; T_{1}\right]$, откуда, учитьвая положительность $f_{i}^{\prime}(0, k)$, заключаем, что $f_{i}^{\prime}(0, k)>0$ на $\left(0 ; T_{1}\right]$.

Далее, из (6) вследствие убывания функции $u_{i}$ имеем $f_{i}(x, k)<f_{i}^{\prime}\left(T_{1}, k\right) u_{i}(x, k)$, откуда следует вторая часть утверждения леммы.

ДоКАЗАТЕЛЬСТво ТЕОРЕмЫ 2. Допустим, что существуют последовательность $k_{j} \rightarrow \infty$ и постоянная $\delta>0$ такие, что $\lambda_{i+1}\left(k_{j}\right)-\lambda_{i}\left(k_{j}\right) \leqslant e^{-\delta k_{j}}$. Имеем

$$
\begin{aligned}
f_{i+1}\left(x, k_{j}\right)= & f_{i}\left(x, k_{j}\right)+\frac{\lambda_{i+1}\left(k_{j}\right)-\lambda_{i}\left(k_{j}\right)}{f_{i}^{\prime}\left(T_{1}, k_{j}\right)} \\
& \times \int_{0}^{x}\left(f_{i}\left(x, k_{j}\right) u_{i}\left(t, k_{j}\right)-u\left(x, k_{j}\right) f_{i}\left(t, k_{j}\right)\right) f_{i+1}\left(t, k_{j}\right) d t .
\end{aligned}
$$

Тогда функция $\tilde{f}_{i+1}=f_{i+1} / f_{i}$ удовлетворяет уравнению

$$
\tilde{f}_{i+1}\left(x, k_{j}\right)=1+\int_{0}^{x} G(x, t, j) \tilde{f}_{i+1}\left(t, k_{j}\right) d t
$$

где

$$
G(x, t, j)=\left(\lambda_{i+1}\left(k_{j}\right)-\lambda_{i}\left(k_{j}\right)\right)\left[\frac{u_{i}\left(t, k_{j}\right) f_{i}\left(t, k_{j}\right)}{f_{i}^{\prime}\left(T_{1}, k_{j}\right)}-\frac{u_{i}\left(x, k_{j}\right) f_{i}^{2}\left(t, k_{j}\right)}{f_{i}\left(x, k_{j}\right) f_{i}^{\prime}\left(T_{1}, k_{j}\right)}\right] .
$$

Используя леммы 2 и 4 , имеем $\left|G\left(x, t, k_{j}\right)\right| \leqslant 2 e^{-\delta k_{j}}$, так что норма в $C\left[0 ; T_{1}\left(k_{j}\right)\right]$ интегрального оператора в правой части последнего уравнения допускает оценку $O\left(e^{-\delta k_{j}}\right)$. Следовательно, $\tilde{f}_{i+1}=1+O\left(e^{-\delta k_{j}}\right), j \rightarrow \infty$, поэтому

$$
f_{i+1}\left(x, k_{j}\right)=f_{i}\left(x, k_{j}\right)\left(1+O\left(e^{-\delta k_{j}}\right)\right), \quad j \rightarrow \infty
$$

равномерно по $x \in\left[0 ; T_{1}\left(k_{j}\right)\right]$. Далее, при $x \in\left[T_{1}\left(k_{j}\right) ; T_{2}\left(k_{j}\right)\right]$ имеем

$$
\begin{aligned}
f_{i+1}\left(x, k_{j}\right)= & f_{i}\left(x, k_{j}\right)+\left(\lambda_{i+1}\left(k_{j}\right)-\lambda_{i}\left(k_{j}\right)\right) \\
& \times \int_{T_{1}\left(k_{j}\right)}^{x}\left(u_{i}\left(x, k_{j}\right) v_{i}\left(t, k_{j}\right)-v\left(x, k_{j}\right) u_{i}\left(t, k_{j}\right)\right) f_{i+1}\left(t, k_{j}\right) d t
\end{aligned}
$$


Повторяя предыдущие рассуждения для последнего уравнения и учитывая при этом утверждение леммы 3 , будем иметь

$$
f_{i+1}\left(x, k_{j}\right)=f_{i}\left(x, k_{j}\right)+O\left(e^{-\delta^{\prime} k_{j}}\right), \quad j \rightarrow \infty,
$$

равномерно по $x \in\left[T_{1}\left(k_{j}\right) ; T_{2}\left(k_{j}\right)\right]$ с некоторым $\delta^{\prime}<\delta$.

Далее, замечая, что оценка (7) справедлива и для отрезка $\left[T_{2}\left(k_{j}\right) ; 1\right]$, получим

$$
f_{i+1}\left(x, k_{j}\right)=f_{i}\left(x, k_{j}\right)+O\left(e^{-\delta^{\prime} k_{j}}\right), \quad j \rightarrow \infty,
$$

равномерно по $x \in[0 ; 1]$. Из этого соотношения видно, что

$$
\left(f_{i+1}, f_{i}\right)=1+O\left(e^{-\delta^{\prime} k_{j}}\right), \quad j \rightarrow \infty,
$$

вопреки тому, что $\left(f_{i+1}, f_{i}\right)=0$. Тем самым теорема доказана.

3. Оператор $L(k)$ с несколькими потенциальными ямами. При доказательстве п. б) леммы 1 мы воспользовались тем, что вне некоторой окрестности точки $a$ собственные функции гармонического осциллятора экспоненциально малы. Это свойство справедливо для оператора с произвольным конечньп числом ям.

Лемма 5. Пусть функиия $q(x) \in C^{(2)}[0 ; 1] u \min _{[0 ; 1]} q(x)=0$. Далее пусть $q(x)>0$ внутри некоторого интервала $(a ; b)$ из $[0 ; 1]$.Тогда для любой нормированной функиии $f_{i}(x, k)$ оператора $L(k)$ и любого отрезка $[\alpha ; \beta]$ из интервала $(a ; b)$ найдется не зависящее от $k$ число $\delta>0$ такое, что

$$
f^{(j)}(x, k)=O\left(e^{-\delta k}\right), \quad k \rightarrow \infty, \quad j=0,1,
$$

равномерно по $x \in[\alpha ; \beta]$.

ДокАЗАТЕльство. Обозначим $m=\min _{[\alpha ; \beta]} q(x)$. Тогда найдется такое положительное число $\varepsilon$, что $q(x)>m / 2$ при $x \in[\alpha-\varepsilon ; \beta+\varepsilon]$. Введем в рассмотрение функцию $p(x)$, обладающую следующими свойствами:

a) $p \in C^{2}[0 ; 1]$;

б) $p(x)=q(x)$ при $x \in[\alpha-\varepsilon ; \beta+\varepsilon]$;

в) $p(x) \geqslant m / 2$ для всех $x \in[0 ; 1]$.

Пусть $M(k)$ - оператор, который получается из $L(k)$ заменой $q(x)$ на $p(x)$. Тогда если $\lambda_{i}(k)$ - собственное значение $L(k)$, соответствующее $f_{i}(x, k)$, то

$$
f_{i}(x, k)=\left(M(k)-\lambda_{i}(k)\right)^{-1} V f_{i},
$$

где $V=p(x)-q(x)$. Согласно пункту б) леммы $1 \lambda_{i}(k) \leqslant m / 4$ при достаточно больших $k>0$. Поэтому, используя ВКБ-разложения [5, гл. II, $\S 2]$, для ядра резольвенты в правой части (8) будем иметь

$$
\frac{\partial^{j}}{\partial x^{j}}(G(x, t, k))=O\left(k^{j-1} e^{-k\left|\int_{t}^{x} \sqrt{p-\lambda_{i}(k)} d \tau\right|}\right), \quad k \rightarrow \infty, \quad j=0,1,
$$

равномерно по $x, t \in[0 ; 1]$. Подставляя (9) при $j=0$ в (8) и учитьвая, что $V=0$ на $[\alpha-\varepsilon ; \beta+\varepsilon]$, получим $f_{i}=O\left(e^{-\delta k}\right), k \rightarrow \infty$, где $\delta=\varepsilon \sqrt{m} / 2$. Далее, чтобы получить оценку для $f_{i}^{\prime}(x, k)$, продифференцируем (8) и подставим в полученное равенство (9) при $j=1$. Лемма доказана.

Следующее утверждение позволяет свести вопрос об асимптотике собственных значений $L(k)$ с произвольным числом ям к случаю одной ямы. 
Лемма 6. Пусть q имеет п потенциальных ям. Тогда для любого собственного значения $\lambda_{j}(k)$ оператора $L(k)$ найдется положительное число $\delta_{j}$ такое, что расстояние от $\lambda_{j}(k)$ до обгединения спектров операторов $L_{i}(k)$ удовлетворяет оиенке

$$
d_{j}(k) \equiv \operatorname{dist}\left(\lambda_{j}(k), \bigcup_{i=1}^{n} \sigma_{i}(k)\right)=O\left(e^{-\delta_{j} k}\right), \quad k \rightarrow \infty
$$

ДокАЗАТЕЛЬСТво. Предположим противное. Пусть для некоторого фиксированного $j$ существуют последовательности $\left\{k_{l}\right\}$ и $\left\{\gamma_{l}\right\}$ такие, что $k_{l} \rightarrow+\infty, \gamma_{l} \rightarrow+0$ и

$$
d_{j}\left(k_{l}\right) \geqslant e^{-\gamma_{l} k_{l}}
$$

Обозначим через $f_{j}(x, k)$ нормированную собственную функцию, соответствующую $\lambda_{j}(k)$. Согласно лемме 5 для любого $i=1, \ldots, n$ существуют положительные числа $\varepsilon_{i}$ и $\tau_{i}$ такие, что

$$
f_{j}=O\left(e^{-\tau_{i} k}\right)
$$

при $k \rightarrow \infty$ равномерно по $x \in\left[b_{i-1} ; b_{i}+\varepsilon_{i}\right] \cup\left[b_{i}-\varepsilon_{i} ; b_{i}\right]$. Пусть $\chi_{i}-$ гладкая функция, равная 1 на $\left[b_{i-1}+\varepsilon_{i} ; b_{i}-\varepsilon_{i}\right]$ и 0 вне $\left(b_{i-1}+\varepsilon_{i} / 2 ; b_{i}-\varepsilon_{i} / 2\right)$. Положим $g_{i}=f_{j} \chi_{i}$, $x \in\left[b_{i-1} ; b_{i}\right]$. Тогда $g_{i} \in D\left(L_{i}\right)$ и

$$
L_{i} g_{i}=\lambda_{j} g_{i}+O\left(e^{-\tau_{i} k}\right), \quad k \rightarrow \infty
$$

равномерно по $x \in\left[b_{i-1} ; b_{i}\right]$. Из (10) следует, что

$$
\left\|\left(L_{i}\left(k_{l}\right)-\lambda\left(k_{l}\right)\right)^{-1}\right\|=O\left(e^{\gamma_{l} k_{l}}\right), \quad l \rightarrow \infty
$$

Следовательно, норма функции $g_{i}\left(x, k_{l}\right)$ в пространстве $L^{2}\left[b_{i-1} ; b_{i}\right]$ при больших $l$ имеет порядок $O\left(e^{-\tau_{i}^{\prime} k_{l}}\right)$ с некоторым $\tau_{i}^{\prime} \in\left(0 ; \tau_{i}\right)$. Отсюда, поскольку $i$ любое, мы можем заключить, что $L^{2}[0 ; 1]$-норма $f_{j}\left(x, k_{l}\right)$ при больших $l$ удовлетворяет оценке $O\left(e^{-\tau k_{l}}\right)$ с некоторым $\tau>0$, что противоречит тому, что $\left\|f_{j}\right\|=1$. Лемма доказана.

Подготовку доказательства теоремы 1 завершает

ЛЕмма 7. Допустим, что q имеет п потенциальных ям. Тогда если некоторые собственные значения $\lambda_{1}(k), \ldots, \lambda_{r}(k)$ оператора $L(k)$ таковь, что для некоторой последовательности $k_{j} \rightarrow+\infty$ и положстельной постоянной $\delta>0$

$$
\lambda_{i}\left(k_{j}\right)-\lambda_{m}\left(k_{j}\right)=O\left(e^{-\delta k_{j}}\right), \quad i, m=1, \ldots, r
$$

$m o r \leqslant n$. 
ДокАЗАТЕЛЬСТво проведем индукцией по $n$. При $n=1$ утверждение леммы следует из теоремы 2. Пусть для $(n-1)$-й ямы утверждение леммы верно. Допустим, что $q$ имеет $n$ потенциальньх ям, а оператор $L(k)$ имеет $n+1$ собственных значений $\lambda_{1}(k), \ldots, \lambda_{n+1}(k)$, экспоненциально близких по некоторой последовательности $k_{j} \rightarrow$ $+\infty$. Обозначим через $f_{1}(x, k), \ldots, f_{n+1}(x, k)$ соответствующие нормированные собственные функции. Тогда для некоторой $m$-й ямы существует $s \geqslant 2$ функций $f_{i_{1}}(x, k), \ldots$, $f_{i_{s}}(x, k)$, удовлетворяющих условию

$$
\left\|f_{i_{l}}\left(x, k_{j}^{\prime}\right)\right\|_{m} \geqslant e^{-\delta_{j} k_{j}^{\prime}}, \quad l=1, \ldots, s
$$

где $\left\{k_{j}^{\prime}\right\}$ - некоторая подпоследовательность последовательности $\left\{k_{j}\right\},\left\{\delta_{j}\right\}$ не зависит от $l$ и $\delta_{j} \rightarrow+0$, a $\|\cdot\|_{m}$ означает норму в $L^{2}\left[b_{m-1} ; b_{m}\right]$. Можно считать (перенумеровав при необходимости), что условию (11) удовлетворяют функции $f_{1}(x, k), \ldots, f_{s}(x, k)$. Также можно считать, что для всех остальных функций справедлива оценка

$$
\left\|f_{i}\left(x, k_{j}^{\prime}\right)\right\|_{m} \leqslant C e^{-\delta^{\prime} k_{j}^{\prime}}, \quad l=s+1, \ldots, n+1
$$

где $C>0, \delta^{\prime}>0$ не зависят от $l, j$.

Действительно, если для $f_{p}(p>s)$ не выполняется (12), то $f_{p}$ будет удовлетворять (11) с некоторьми $\left\{\delta_{j}^{\prime}\right\}$ и $\left\{k_{j}^{\prime \prime}\right\}$ - подпоследовательностью $\left\{k_{j}^{\prime}\right\}$. Тогда $f_{p}$ можно присоединить к первой группе, заменяя при этом $\left\{k_{j}^{\prime}\right\}$ на $\left\{k_{j}^{\prime \prime}\right\}$ и $\delta_{j}$ на $\delta_{j}^{\prime \prime}=\max \left\{\delta_{j}, \delta_{j}^{\prime}\right\}$.

Докажем, что для функций $f_{2}, \ldots, f_{s}$ (всюду в дальнейшем под $f_{i}$ будем понимать $\left.f_{i}\left(x, k_{j}^{\prime}\right)\right)$ найдется некоторое положительное число $\alpha$ такое, что

$$
\left|\left(f_{1}, f_{i}\right)_{m}\right| \geqslant\left\|f_{1}\right\|_{m}\left\|f_{i}\right\|_{m}\left(1-e^{-\alpha k_{j}^{\prime}}\right), \quad i=2, \ldots, s
$$

где $(\cdot, \cdot)_{m}$ означает скалярное произведение в $L^{2}\left[b_{m-1} ; b_{m}\right]$.

Предположим противное. Это означает, что для некоторой функции $f_{p}, 2 \leqslant p \leqslant s$, найдется подпоследовательность последовательности $\left\{k_{j}^{\prime}\right\}$, которую снова обозначим $\left\{k_{j}^{\prime}\right\}$, и последовательность $\left\{\alpha_{j}\right\}: \alpha_{j} \rightarrow+0, j \rightarrow \infty$, удовлетворяющие условию

$$
\left|\left(f_{1}, f_{p}\right)_{m}\right|<\left\|f_{1}\right\|_{m}\left\|f_{p}\right\|_{m}\left(1-e^{-\alpha_{j} k_{j}^{\prime}}\right)
$$

Тогда, умножая функции

$$
f_{1} \quad \text { и } g=f_{p}-\frac{\left(f_{1}, f_{p}\right)_{m}}{\left\|f_{1}\right\|_{m}^{2}} f_{1}
$$

на срезающую функцию $\chi_{m}(x)$, введенную при доказательстве леммы 6 , пронормировав полученные функции и учитьвая при этом, что согласно (11) и (14) $\|g\|_{m} \geqslant$ $e^{-\left(\delta_{j}+\alpha_{j}\right) k_{j}^{\prime}}$, построим две функции $\tilde{f}_{1}$ и $\tilde{g}$, обладающие следующими свойствами: $\tilde{f}_{1}$ и $\tilde{g} \in D\left(L_{m}\right),\left\|\tilde{f}_{1}\right\|_{m}=\|\tilde{g}\|_{m}=1,\left(\tilde{f}_{1}, \tilde{g}\right)_{m}=0$ и

$$
\left\|\left(L_{m}\left(k_{j}^{\prime}\right)-\lambda_{1}\left(k_{j}^{\prime}\right)\right) \tilde{f}_{1}\right\|_{m} \leqslant e^{-d k_{j}^{\prime}}, \quad\left\|\left(L_{m}\left(k_{j}^{\prime}\right)-\lambda_{1}\left(k_{j}^{\prime}\right)\right) \tilde{g}\right\|_{m} \leqslant e^{-d k_{j}^{\prime}}, \quad d>0 .
$$

Отсюда в силу известной вариационной теоремы (см., например, [6, гл. VI, п. 82, теорема 1] на отрезке $\left[\lambda_{1}\left(k_{j}^{\prime}\right)-e^{-d k_{j}^{\prime}}, \lambda_{1}\left(k_{j}^{\prime}\right)+e^{-d k_{j}^{\prime}}\right]$ существуют по крайней мере 2 
собственных значения оператора $L\left(k_{j}^{\prime}\right)$, что противоречит теореме 2 . Тем самым (13) установлено.

Введем в рассмотрение функции $\tilde{f}_{i}=g_{i} /\left\|g_{i}\right\|, i=2, \ldots, s$, где

$$
g_{i}=f_{i}-\frac{\left(f_{1}, f_{i}\right)_{m}}{\left\|f_{1}\right\|_{m}^{2}} f_{1} .
$$

Используя (13), легко проверить, что для $\tilde{f}_{i}$

$$
\left\|\tilde{f}_{i}\right\|_{m} \leqslant 2 e^{-\alpha k_{j}^{\prime}}, \quad i=2, \ldots, s .
$$

Рассмотрим теперь оператор $\widetilde{L}(k)$, который получается из $L(k)$ заменой $q$ на $\tilde{q}$, где $\tilde{q}$ - дважды непрерывно дифференцируемая функция, совпадающая с $q$ вне $\left(b_{m-1} ; b_{m}\right)$ и удовлетворяющая на $\left[b_{m-1} ; b_{m}\right]$ неравенству $\tilde{q}(x) \geqslant q_{0}, q_{0}$ - положительная постоянная ("засьпали" $m$-ю яму). Тогда, воспользовавшись неравенствами (12) и (15), будем иметь

$$
\begin{array}{ll}
\left\|\left(\widetilde{L}\left(k_{j}^{\prime}\right)-\lambda_{i}\left(k_{j}^{\prime}\right)\right) \tilde{f}_{i}\right\|_{m} \leqslant e^{-\beta k_{j}^{\prime}}, & i=2, \ldots, s, \\
\left\|\left(\widetilde{L}\left(k_{j}^{\prime}\right)-\lambda_{i}\left(k_{j}^{\prime}\right)\right) f_{i}\right\|_{m} \leqslant e^{-\beta k_{j}^{\prime}}, \quad i=s+1, \ldots, n+1,
\end{array}
$$

с некоторым $\beta>0$. Отсюда, учитьвая, что $\tilde{f}_{2}, \ldots, \tilde{f}_{s}, f_{s+1}, \ldots, f_{n+1}$ образуют ортонормированную систему, заключаем, что оператор $\widetilde{L}\left(k_{j}^{\prime}\right)$ с $n-1$ потенциальными ямами имеет $n$ экспоненциально близких собственных значений, что противоречит допущению индукции. Тем самьм лемма доказана.

4. ДоКАЗАТЕЛЬСТво ТЕОРЕмЫ 1. Достаточность. Пусть $\mu_{i}(k)$ - собственные значения $L_{i}(k)$ такие, что $\mu_{i}(k)-\mu_{j}(k)=O\left(e^{-\delta k}\right), k \rightarrow \infty, i, j=1, \ldots, n$, при некотором $\delta>0$. Обозначим через $f_{i}(x, k)$ соответствуюшие нормированные собственные функции. Умножив $f_{i}$, как и при доказательстве леммы 6 , на соответствующую срезающую функцию $\chi_{i}$ и продолжив ее нулем на $[0 ; 1] \backslash\left(b_{i-1} ; b_{i}\right)$, а затем пронормировав, получим функцию $\tilde{f}_{i}$, обладающую следующими свойствами: $\left\|\tilde{f}_{i}\right\|=1, L \tilde{f}_{i}=\mu_{i}(k) \tilde{f}_{i}+O\left(e^{-\delta_{i} k}\right)$, $k \rightarrow \infty, \delta_{i}>0$, равномерно по $x \in[0 ; 1]$. Отсюда, применяя вариационную теорему, заключаем, что существуют $\delta_{0}>0$ и $K>0$ такие, что при всех $k \geqslant K$ в отрезке $I(k)=\left[\mu_{1}(k)-e^{-\delta_{0} k} ; \mu_{1}(k)+e^{-\delta_{0} k}\right]$ содержится не менее $n$ собственных значений оператора $L(k)$. Тогда согласно лемме 7 при достаточно больших $k$ оператор $L(k)$ имеет в отрезке $I(k)$ ровно $n$ собственных значений $\lambda_{m(k)}(k), \ldots, \lambda_{m(k)+n-1}(k)$. Покажем, что при достаточно больших $k$ номера собственных значений, попавших в отрезок $I(k)$, не зависят от $k$. Допустим противное. Это означает, что найдется некоторая последовательность $\left\{k_{j}\right\}$ такая, что $k_{j} \rightarrow \infty$ и $m(k)$ терпит скачок в точках $k_{j}$. Тогда поскольку собственные значения оператора $L(k)$ непрерьвны при $k>0$ (лемма 1 ), то $I\left(k_{j}\right)$ содержит ровно $n+1$ точек спектра оператора $L\left(k_{j}\right)$, что невозможно в силу леммы 7 .

Необходимость. Пусть оператор $L(k)$ с $n$ потенциальньми ямами имеет $n$ собственных чисел $\lambda_{1}(k), \ldots, \lambda_{n}(k)$, экспоненциально близких на бесконечности. Сначала докажем, что для всех $m=1, \ldots, n$ найдутся положительные числа $\delta_{m}$ и $K_{m}$ такие, что при всех $k \geqslant K_{m}$ в отрезке $I_{m}(k)=\left[\lambda_{1}(k)-e^{-\delta_{m} k} ; \lambda_{1}(k)+e^{\delta_{m} k}\right]$ сушествуют собственные значения оператора $L_{m}(k)$. Допустим, что это не так, т.е. существует такое $1 \leqslant m \leqslant n$, что

$$
\operatorname{dist}\left\{\lambda_{1}\left(k_{j}\right) ; \sigma_{m}\left(k_{j}\right)\right\} \geqslant C_{1} e^{-\alpha_{j} k_{j}}
$$


для некоторых последовательностей $k_{j} \rightarrow+\infty$ и $\alpha_{j} \rightarrow+0$ и положительной постоянной $C_{1}$. Так как по условию $\lambda_{1}(k), \ldots, \lambda_{n}(k)$ экспоненциально близки на бесконечности, соотношение (16) верно для всех $\lambda_{l}\left(k_{j}\right), l=1, \ldots, n$ (возможно с другими значениями $\left.C_{l}>0\right)$. Отсюда следует, что

$$
\left\|\left(L_{m}\left(k_{j}\right)-\lambda_{l}\left(k_{j}\right)\right)^{-1}\right\|=O\left(e^{\alpha_{j} k_{j}}\right), \quad j \rightarrow \infty, \quad l=1, \ldots, n .
$$

Рассуждая теперь так же, как при доказательстве леммы 6, заключаем, что для нормированных собственных функций $f_{l}(x, k)$ справедлива равномерная по $x \in\left[b_{m-1} ; b_{m}\right]$ оценка

$$
f_{l}\left(x, k_{j}\right)=O\left(e^{-\delta k_{j}}\right), \quad j \rightarrow \infty, \quad l=1, \ldots, n,
$$

с некоторым $\delta>0$.

Рассмотрим оператор $\widetilde{L}(k)$, введенный при доказательстве леммы 7 . Из соотношения (17) имеем

$$
\left\|\left(\widetilde{L}\left(k_{j}\right)-\lambda_{l}\left(k_{j}\right)\right) f_{l}\left(x, k_{j}\right)\right\|=O\left(e^{-\delta k_{j}}\right), \quad j \rightarrow \infty, \quad l=1, \ldots, n,
$$

так что оператор $\widetilde{L}\left(k_{j}\right)$ с $n-1$ потенциальными ямами имеет $n$ собственных чисел, экспоненциально близких на бесконечности, что невозможно в силу леммы 7. Следовательно, при любом $m=1, \ldots, n$ отрезок $I_{m}(k)$ при достаточно больших $k>0$ содержит хотя бы одно собственное значение оператора $L_{m}(k)$. Применяя теперь теорему 2 , мы можем утверждать, что в $I_{m}(k)$ при достаточно больших $k>0$ содержится единственная точка спектра $L_{m}(k)$. Обозначим ее $\mu_{m(k)}(k)$. Рассуждая далее так же, как при доказательстве достаточной части теоремы 1 , получим, что номер $m(k)$ не зависит от $k$ при достаточно больших $k>0$. Теорема доказана.

5. Анализ условий расщепления спектра. Пример. В этом пункте мы дадим некоторое простое достаточное условие существования экспоненциально близких собственных значений операторов $L_{i}(k)$. В конце пункта построен пример, показьвающий, что это условие не является необходимым.

Tеорема 3. Пусть $q \in C^{2}[0 ; 1]$ и в $(0 ; 1)$ существуют $n$ точек $0<a_{1}<\cdots<$ $a_{n}<1$, удовлетворяющих условиям 1)-2). Далее, пусть существует $\varepsilon>0$ такое, что

$$
q\left(x+a_{i}-a_{1}\right) \equiv q(x), \quad x \in\left[a_{1}-\varepsilon ; a_{1}+\varepsilon\right], \quad i=2, \ldots, n .
$$

Тогда операторы $L_{1}(k), \ldots, L_{n}(k)$ имеют собственные значения $\mu_{1}(k), \ldots, \mu_{n}(k)$, экспоненииально близкие на бесконечности.

Доказательству теоремы предпошлем две леммы.

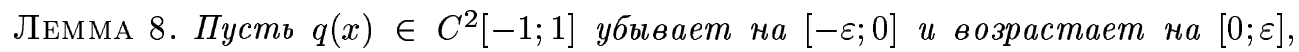
$0<\varepsilon<1$. Далее, пусть $V \in L^{\infty}[-1 ; 1], V=0$ в некоторой окрестности точки 0 и неотрицательна на остальной части $[-1 ; 1]$. Обозначим через $\left\{\lambda_{i}(k)\right\} u\left\{\mu_{i}(k)\right\}$ собственные значения операторов $L(k)$ и $M(k)=L(k)+V$, занумерованные в порядке возрастания. Тогда

$$
\lambda_{i}(k)-\mu_{i}(k)=O\left(e^{-\delta_{i} k}\right), \quad k \rightarrow \infty
$$

где $\delta_{i}>0$ не зависят от $k$. 
ДокАЗАТЕЛЬСтво. Так как $V \geqslant 0$, то $\mu_{i}(k) \geqslant \lambda_{i}(k)$. Пусть $f_{i}(x, k)-$ нормированная собственная функция $L(k)$, соответствующая $\lambda_{i}(k)$. Согласно лемме 5 имеем $\left(M(k) f_{i}, f_{i}\right)=\lambda_{i}(k)+\left(V f_{i}, f_{i}\right)=\lambda_{i}(k)+O\left(e^{-\delta_{i} k}\right), k \rightarrow \infty, \delta_{i}>0$. Отсюда в силу принципа минимакса $\left[2, \S\right.$ XII.1, теорема XIII.1] $\mu_{i}(k) \leqslant \lambda_{i}(k)+C_{i} e^{-\delta_{i} k}$, где $C_{i}>0$ не зависит от $k$.

Лемма 9. Пусть $q(x) \in C^{2}[-1 ; 1], q(0)=0, q(x)>0$ nри $x \neq 0, q$ убывает на $(-\varepsilon ; 0)$ и возрастает на $(0 ; \varepsilon), 0<\varepsilon<1$. Далее, пусть $\left(a_{1} ; b_{1}\right) u\left(a_{2} ; b_{2}\right)-$ произвольные интерваль из $(-1 ; 1)$, содержащие точку 0 . Обозначим $L_{i}(k), i=1,2$, самосопряженные операторы, порожденные выражением $l(k)$ в $L^{2}\left(a_{i} ; b_{i}\right)$ и краевыми условиями $y\left(a_{i}\right)=y\left(b_{i}\right)=0$. Тогда для собственных значений $\left\{\lambda_{j}^{i}(k)\right\}$ операторов $L_{i}(k)$ при каждом $j=1,2, \ldots$ имеют место асимптотические соотношения

$$
\lambda_{j}^{1}(k)-\lambda_{j}^{2}(k)=O\left(e^{-\delta_{j} k}\right), \quad k \rightarrow \infty,
$$

с некоторыми $\delta_{j}>0$, не зависящими от $k$.

ДоказАтельство. Обозначим $a=\min \left\{a_{1}, a_{2}\right\}, A=\max \left\{a_{1}, a_{2}\right\}, b=\max \left\{b_{1}, b_{2}\right\}$, $B=\min \left\{b_{1}, b_{2}\right\}$. Пусть $M_{1}(k)$ и $M_{2}(k)$ - операторы, соответствуюшие отрезкам $[a ; b]$ и $[A ; B],\left\{\mu_{j}^{1}(k)\right\}$ и $\left\{\mu_{j}^{2}(k)\right\}$ - их спектры. Имеем $\mu_{j}^{1}(k) \leqslant \lambda_{j}^{i}(k) \leqslant \mu_{j}^{2}(k), i=1,2$. Пусть $f_{j}(x, k)$ - нормированные собственные функции $M_{1}(k)$, соответствующие $\mu_{j}^{1}(k)$. Применяя лемму 5 , так же, как и при доказательстве леммы 6, построим функции $\tilde{f}_{j}(x, k)$, обладающие следующими свойствами:

$\tilde{f}_{j} \in D\left(M_{2}\right), \quad\left\|\tilde{f}_{j}\right\|_{[A ; B]}=1, \quad\left\|\left(M_{2}-\mu_{j}^{1}\right) \tilde{f}_{j}\right\|_{[A ; B]}=O\left(e^{-\delta_{j} k}\right), \quad k \rightarrow \infty, \quad \delta_{j}>0$, где $\|\cdot\|_{[A ; B]}$ Означает норму в $L^{2}[A ; B]$. Отсюда следует, что

$$
\mu_{j}^{2}(k) \leqslant \mu_{j}^{1}(k)+C_{j} e^{-\delta_{j} k}, \quad C_{j}>0,
$$

что и доказьвает лемму.

ДоКАЗАТЕЛЬСТВО ТЕОРЕМЫ 3. Из условий теоремы вытекает, что существуют функция $p(x) \in C^{2}[-1 ; 1]$ и положительная постоянная $\varepsilon^{\prime}<\varepsilon$, которые удовлетворяют следующим условиям: $p(x)=q\left(x-a_{1}\right),|x| \leqslant \varepsilon^{\prime}, q(x) \geqslant p\left(x+a_{i}\right), x \in\left[b_{i-1} ; b_{i}\right]$, $i=1, \ldots, n$.

Обозначим через $M_{i}(k)$ операторы, которые получаются из $L_{i}(k)$ заменой $q(x)$ на $p\left(x+a_{i}\right)$. Из леммы 8 следует, что собственные значения операторов $M_{i}(k)$ и $L_{i}(k)$ с одинаковыми номерами экспоненциально близки на бесконечности. Из леммы 9 следует, что операторы $M_{1}(k), \ldots, M_{n}(k)$ имеют собственные значения, экспоненциально близкие на бесконечности. Отсюда получаем утверждение теоремы.

ЗАмечание 1 . Так как задача (1)-(2) на отрезке [-a; $a$ с четным потенциалом инвариантна относительно замены $x$ на $-x$, утверждение теоремы 3 остается в силе, если равенства (18) при некоторых $i=2, \ldots, n$ заменить на равенства $q\left(x+a_{i}-a_{1}\right)=q\left(2 a_{1}-x\right)$.

ЗАмЕчАниЕ 2. Очевидно, все утверждения справедливы и в том случае, когда функция $q(x)$ имеет конечное число точек разрыва $r_{1}, \ldots, r_{s}$, отличных от точек $a_{i}$, так что $q \in C^{2}\left[r_{i-1} ; r_{i}\right], i=1, \ldots, s+1$, где $r_{0}=0, r_{s+1}=1$.

В заключение мы рассмотрим пример, показьвающий, что для существования экспоненциально близких собственных значений операторов $L_{i}(k)$ не обязательно, чтобы ямы были одинаковой формы. 
ПРИмеР. Пусть $a \in(0 ; 1)$. Положим

$$
q(x)= \begin{cases}x, & 0 \leqslant x \leqslant a \\ b(1-x), & a \leqslant x \leqslant 1\end{cases}
$$

и обозначим через $L_{1}(k)$ и $L_{2}(k)$ операторы, порожденные дифференциальньг выражением $-k^{-2} d^{2} / d x^{2}+q(x)$ и нулевыми краевьми условиями в $L^{2}[0 ; a]$ и $L^{2}[a ; 1]$ соответственно. Далее, пусть $M_{1,2}(k)$ - операторы, которые получаются из $L_{1,2}(k)$ заменой $[0 ; a]$ на $[0 ;+\infty)$ и $[a ; 1]$ на $(-\infty ; 1]$. Обозначим через $\nu_{m}^{i}(k)$ и $\mu_{m}^{i}(k), i=1,2$, $m=1,2, \ldots$, собственные значения операторов $M_{i}(k)$ и $L_{i}(k)$. Тогда, повторяя рассуждения, проведенные при доказательстве п. б) леммы 1 , будем иметь

$$
\nu_{m}^{1}(k)=\frac{\xi_{m}}{k^{2 / 3}}, \quad \nu_{m}^{2}(k)=b^{2 / 3} \nu_{m}^{1}(k), \quad \mu_{m}^{1,2}(k)=\nu_{m}^{1,2}(k)+O\left(e^{-\delta k}\right), \quad k \rightarrow \infty
$$

где $-\xi_{m}<0-m$-й нуль функции Эйри, $\delta>0$. Отсюда видно, что если $b=\left(\xi_{m} / \xi_{j}\right)^{3 / 2}$ при некоторых $m, j \in \mathbb{N}$, то $\nu_{m}^{2}(k)=\nu_{j}^{1}(k)$; следовательно, собственные значения $\mu_{j}^{1}(k)$ и $\mu_{m}^{2}(k)$ экспоненциально близки.

\section{СПИСОК ЦИТИРОВАННОЙ ЛИТЕРАТУРЫ}

[1] Аленицын А. Г. Расщепление спектра, порожденное потенциальным барьером в задачах с симметричным потенциалом // Дифференц. уравнения. 1982. Т. 18. №11. С. 1971-1975.

[2] Рид М., Саймон Б. Методы современной математической физики. Т. 4. М.: Мир, 1982.

[3] Хартман Ф. Обыкновенные дифференциальные уравнения. М.: Мир, 1970.

[4] Наймарк М. А. Линейные дифференциальные операторы. М.: Наука, 1969.

[5] Федорюк М. В. Асимптотические методы для линейных обыкновенных дифференциальных уравнений. М.: Наука, 1983.

[6] Ахиезер Н.И., Глазман И. М. Теория линейных операторов в гильбертовом пространстве. Т. 1. Харьков: Вища школа, 1977.

Башкирский государственный университет, г. Уфа

Поступило

E-mail: IshkinHK@bsu.bashedu.ru

14.12 .2000 